\section{Inflammatory State After Intrathoracic Breast Implant Placement for Postpneumonectomy Syndrome}

\author{
Aurelie Merlo, MD, Catharine McDermott, MD, \\ Hadley Wilson, MD, and Benjamin Haithcock, MD
}

Division of Cardiothoracic Surgery, Department of Surgery, University of North Carolina at Chapel Hill, Chapel Hill, North Carolina

We describe a case of a 16-year-old patient who underwent right pneumonectomy for pulmonary vein atresia and developed postpneumonectomy syndrome. She had an $800-\mathrm{cm}^{3}$ saline-filled silicone tissue expander placed in the right hemithorax with resolution of her postpneumonectomy syndrome. However, 2 years later, she developed fevers, night sweats, and arthralgias. Her medical workup was negative for vasculitis, inflammatory bowel disease, and infectious etiologies. She underwent tissue expander removal, resulting in resolution of her symptoms. This report describes a case of an inflammatory state created by a tissue expander placed for postpneumonectomy syndrome.

$\mathrm{P}$ ostpneumonectomy syndrome is a rare complication after pneumonectomy. It occurs when the mediastinum shifts toward the pneumonectomy space, distorting mediastinal structures such as the esophagus and airways ${ }^{1}$ and causing symptoms of dysphagia, respiratory distress, and stridor. There have been numerous techniques described to address this technique, the first of which was described by pediatric surgeons in the treatment of pulmonary agenesis. ${ }^{2}$ Once postpneumonectomy syndrome was described by Wasserman and colleagues, ${ }^{3}$ more attention was brought to this complication, and it was noted to be more common in children and adolescents because of the elasticity of their tissues. ${ }^{4}$ Techniques to address symptoms of compression include division of the ligamentum arteriosum, fixation of the aorta or pulmonary artery to the sternum, and placement of expandable stents without reliability in outcome. ${ }^{4}$ Further advancements were made in the treatment of postpneumonectomy syndrome, and the focus turned to filling the postpneumonectomy space. To this end, methods such as phrenectomy, and placement of prostheses (Lucite plastic balls [Lucite International, Birmingham, UK], silastic implants, and saline-filled breast implants) became the standard of care. ${ }^{4}$ We

Accepted for publication Mar 8, 2020.

Address correspondence to Dr Merlo, University of North Carolina at Chapel Hill, 3040 Burnett-Womack Bldg, CB\# 7065, Chapel Hill, NC 27599-7065; email: aurelie.merlo@unchealth.unc.edu. describe a case of a 16-year-old patient who underwent pneumonectomy for pulmonary vein atresia and developed postpneumonectomy syndrome. She was treated with placement of a tissue expander but then developed silicone incompatibility syndrome requiring removal of the implant.

A 16-year-old girl presented to the Thoracic Surgery Clinic for evaluation of pneumonectomy for right-sided pulmonary vein atresia. She was first noted to have symptoms at 8 months of age, when she presented with increased work of breathing. A computed tomography scan of the chest and echocardiogram were performed which revealed right-sided pulmonary vein atresia (Figure 1). At that time, it was thought that she would eventually require pneumonectomy; however, if pneumonectomy is performed at a young age, mechanical problems such as scoliosis can arise because of the disparity in weight between the right chest and the left chest, and there are higher rates of postpneumonectomy syndrome. ${ }^{1}$ At 16 years of age, she presented with recurrent hemoptysis. At that time, it was decided in a multidisciplinary fashion to pursue workup for rightsided pneumonectomy. A ventilation-perfusion scan was performed, which revealed very limited perfusion of the right lung (Figure 1). Her lung function was adequate with forced expiratory volume in 1 second $\left(\mathrm{FEV}_{1}\right)$ of $86 \%$ predicted and diffusion capacity of the lung for carbon monoxide of $63 \%$ predicted. Given these findings, the patient was deemed to have appropriate pulmonary reserve to tolerate right pneumonectomy. This was attempted with video-assisted thoracoscopic approach, but given the large amount of collateral vessels encountered in the hilum, the decision was made to convert to a vertical muscle-sparing thoracotomy. Larger pulmonary vein collaterals were ligated. The pulmonary artery and right mainstem bronchus were ligated at the hilum, and the lung was removed. The mediastinum was balanced at the conclusion of the case. She was discharged on postoperative day 6 . After her operation, her pneumonectomy space filled up appropriately with fluid.

A year and a half later, the patient was re-referred to our clinic because of worsening shortness of breath. Pulmonary function tests performed 11 months after pneumonectomy demonstrated an $\mathrm{FEV}_{1}$ of $1.34 \mathrm{~L}$, but 6 months after that her $\mathrm{FEV}_{1}$ had decreased to $0.77 \mathrm{~L}$. She was also experiencing lower extremity swelling. Crosssectional computed tomography scan imaging demonstrated left-sided airway narrowing and severe shifting of the mediastinum into the postpneumonectomy space (Figure 2). Her symptoms were consistent with postpneumonectomy syndrome, and she was brought to the operating room for redo thoracotomy and tissue expander placement in the right chest. An $800-\mathrm{mL}$ saline filled breast implant (Natrelle $68 \mathrm{HP}$ [Allergan, Dublin, Ireland]) was placed and filled with $780 \mathrm{~mL}$ of saline. Postoperative imaging demonstrated resolution of right-sided mediastinal shift, and the patient had 


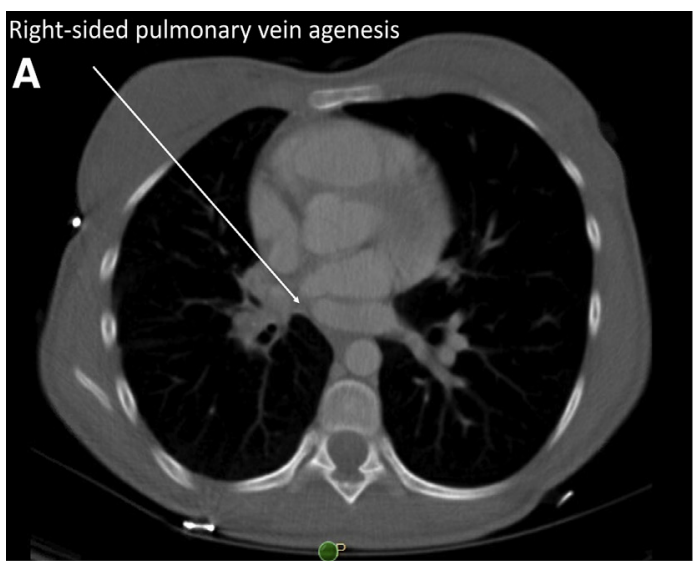

$\mathbf{B}$

\section{Superior lungs}
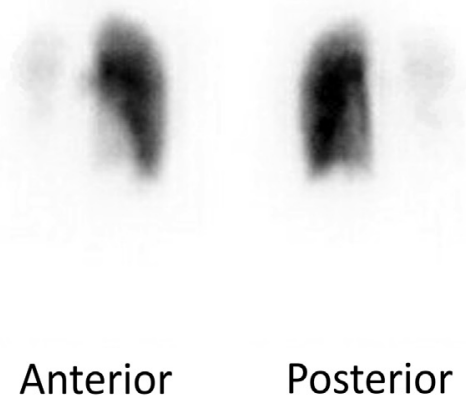

Anterior

Posterior

Figure 1. Prepneumectomy and immediately postpneumonectomic imaging. (A) Preoperative computed tomography scan demonstrating rightsided pulmonary vein atresia. (B) Prepneumonectomy ventilation-perfusion scan with anterior and posterior views demonstrating minimal perfusion to the right lung and homogenous perfusion of the left lung.

improvement in her respiratory symptoms (Figure 2). The patient had visible improvement in airway exam on bronchoscopic evaluation after tissue expander placement (Figure 3). She was discharged on postoperative day 4.

In the 2 years after tissue expander placement, the patient developed systemic joint pain, low-grade fevers, and night sweats, despite no symptoms of dyspnea. She underwent an extensive workup with numerous specialties (gastroenterology, rheumatology, infectious disease, nephrology) to rule out vasculitis, inflammatory bowel disease, and other rheumatologic diseases, which was all negative. After this negative workup, the concern was that the tissue expander was causing an inflammatory state, triggering the patient's symptoms. The patient therefore underwent redo thoracotomy and tissue expander removal. The rind from the tissue expander was left in place and the postpneumonectomy space was instilled with antibiotic solution. The patient did well postoperatively and was discharged on postoperative day 3. Her symptoms of joint pain, fevers, and night sweats resolved. She has not had recurrence of her postpneumonectomy syndrome symptoms.

\section{Comment}

This case report describes an inflammatory state after tissue expander placement in a patient with a history of postpneumonectomy syndrome. There are some reports of development of an inflammatory state existing after placement of tissue expanders for breast reconstruction. ${ }^{5}$ The inflammatory state is thought to be secondary to

Figure 2. Computed tomography scan demonstrating left mainstem bronchus narrowing concerning for postpneumonectomy syndrome. Circles highlight areas of visible airway collapse.

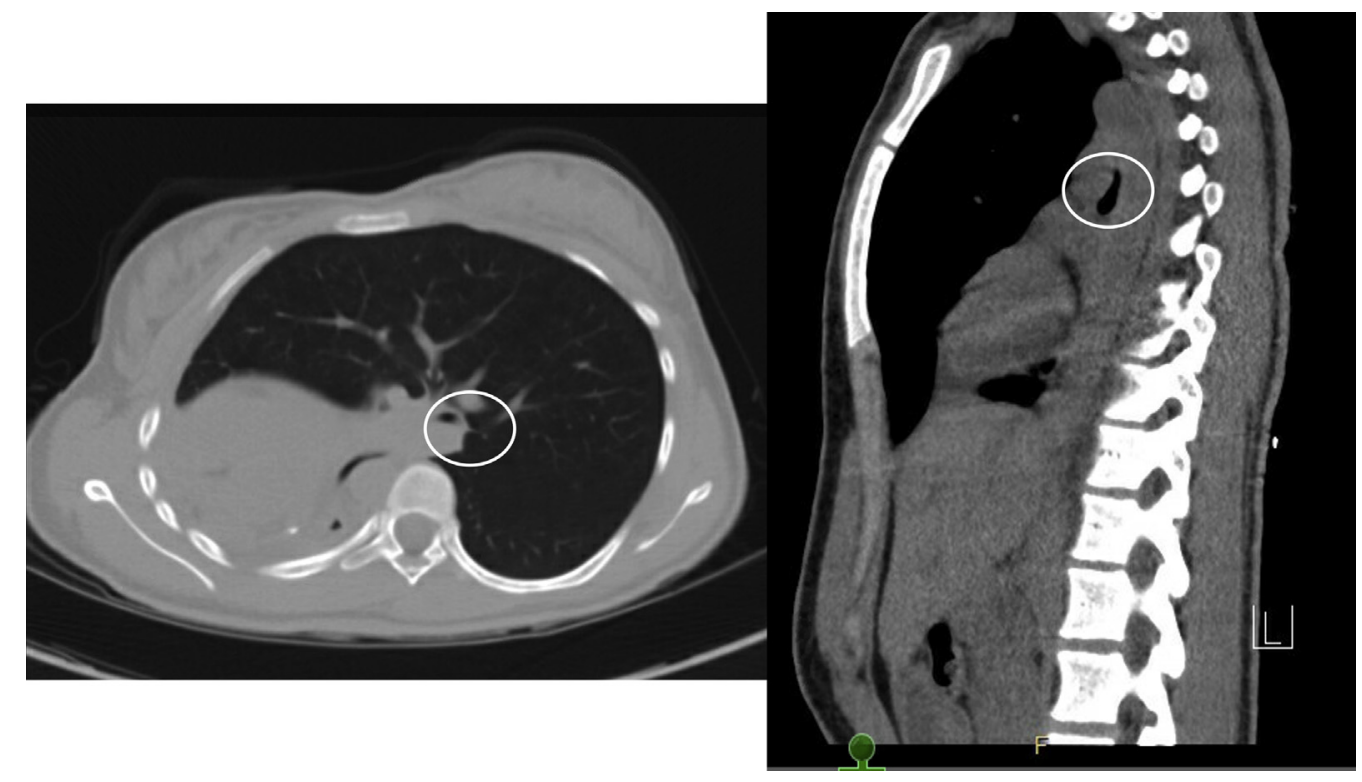




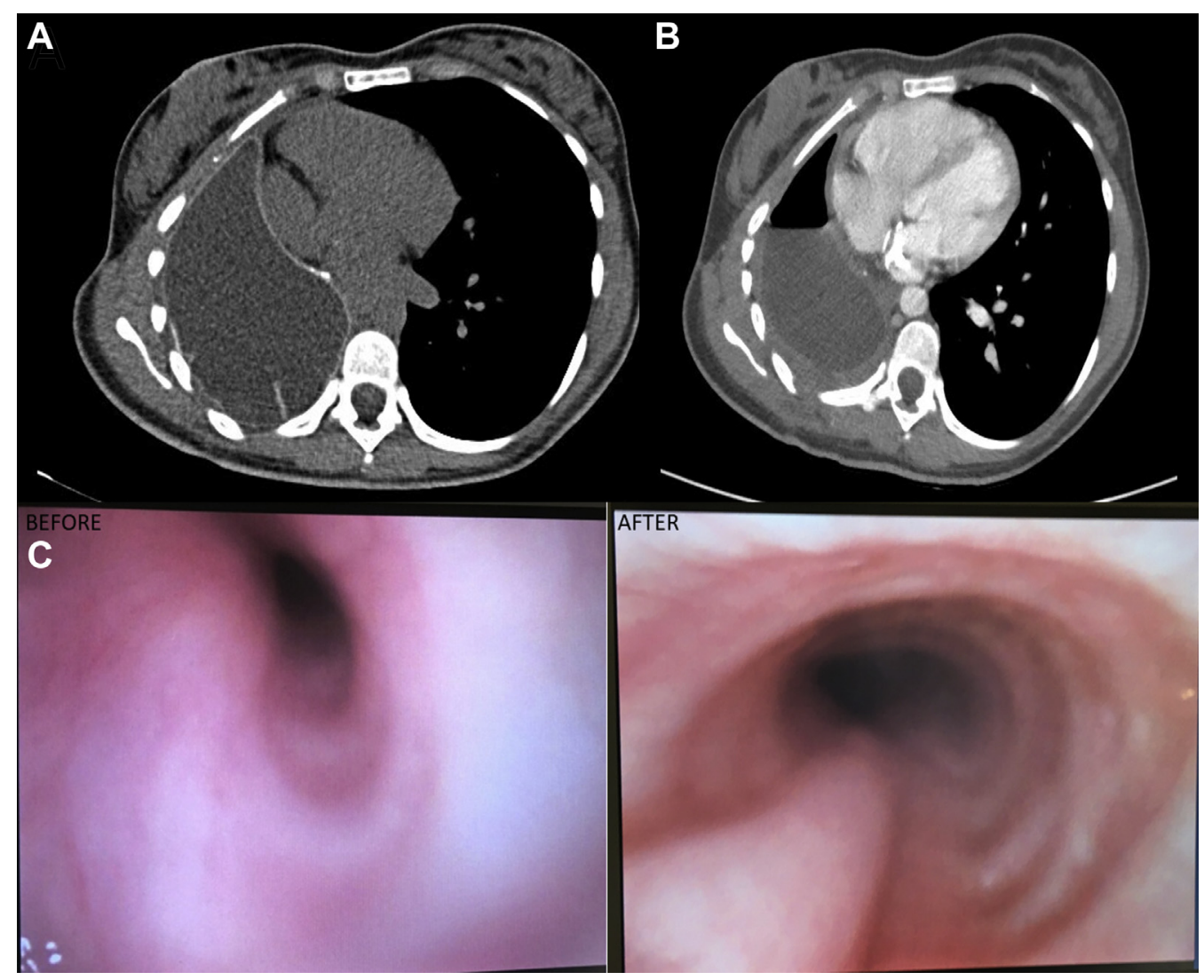

Figure 3. Computed tomography (CT) and bronchoscopy after tissue expander placement and removal. (A) CT scan demonstrating adequate positioning of mediastinal structures after tissue expander placement in the right chest. (B) CT scan of tissue expander removal with antibiotic solution instilled in right hemithorax. (C) Fiberoptic bronchoscopy view of the left main bronchus before (left) and after (right) insertion of the tissue expander.

the use of silicone and has been named silicone incompatibility syndrome or, more broadly, autoimmune or inflammatory syndrome by adjuvants. ${ }^{5}$ The presumed mechanism is silicone-containing particles taken up by macrophages, resulting in cytokine activation and enzyme release. The silicone-containing particles are also taken to the lymph nodes, enhancing the inflammatory state. ${ }^{5}$ The associated symptoms are broad and include chronic fatigue, arthralgias, myalgias, pyrexia, dry mouth, Raynaud's phenomenon, abdominal pain, and cystitis. ${ }^{5}$ Removal of the tissue expander is purported to resolve symptoms in $60 \%$ to $80 \%$ of cases. ${ }^{6}$ Indeed, our patient had resolution of symptoms after implant removal. As this is a rare case, it is difficult to determine the incidence of this complication and optimal management of the postpneumonectomy syndrome patient.

\section{References}

1. Valji AM, Maziak DE, Shamji FM, Matzinger FR. Postpneumonectomy syndrome: recognition and management. Chest. 1998;114:1766-1769.

2. Schaffer AJ, Rider RV. A note on the prognosis of pulmonary agenesis and hypoplasia according to the side affected. J Thorac Surg. 1957;33:379-382.

3. Wasserman K, Jamplis RW, Lash H, Brown HV, Cleary MG, Lafair J. Postpneumonectomy syndrome. Surgical correction using Silastic implants. Chest. 1979;75:78-81.

4. Jung JJ, Cho JH, Kim HK, et al. Management of postpneumonectomy syndrome using tissue expanders. Thorac Cancer. 2016;7:88-93.

5. Cohen Tervaert JW, Colaris MJ, van der Hulst RR. Silicone breast implants and autoimmune rheumatic diseases: myth or reality. Curr Opin Rheumatol. 2017;29:348-354.

6. de Boer M, Colaris M, van der Hulst RRWJ, Cohen Tervaert JW Is explantation of silicone breast implants useful in patients with complaints? Immunol Res. 2017;65:25-36. 